\title{
Foundation Health, Ethics and Body Situations
}

\author{
Bernard T ${ }^{1,3 *}$, Francois Jaco b ${ }^{2}$ and Ogien Ruwen ${ }^{4}$ \\ ${ }^{1,3}$ Department of Art Sciences and Sociology, Pantheon Sorbonne Paris, France \\ ${ }^{2}$ Associate Researcher, Forensic Laboratory, Descartes Paris V, France \\ ${ }^{4}$ Researcher, Department of Contemporary Sociology, Descartes Paris V, France
}

*Corresponding author: Bernard T, Department of Art Sciences and Sociology,

Received Date: December 17, 2018

Pantheon Sorbonne Paris, France.

Published Date: January 08, 2019

\section{Introduction}

We can understand the great complexity that tends to increase with the development of knowledge and technology. In reality, the assembly of knowledge and the evolution of all scientific approaches amplify the science of possibilities by discarding the drive towards a control of medical variability while multiplying, contradictorily, the means of selection and preferences leading to questions. Socrates in his time submitted in one of his aphorisms: "Our uncertainty increases according to knowledge as the perimeter of a circle according to its radius". All primary ethics refer to this text of Spinoza in his Book III and proposals VI, VII and VIII, including: "Everything, as much as it is in it, strives to persevere in its being"1. The many ethical issues between patient/doctor/medicine and other places of contact, including families, courts and judicial institutions, medical or paramedical institutions (Ehpad, physical or neurological care centers) are confronted with new situations, sometimes unwanted and enviable problems. This touches on the limits of ethics in medical art, in the art of verbal communication. The use of medical ethics throughout the world of personal care or maintenance, the accompaniment of vulnerable or simply aging people, in the face of medical, legal and practical contingencies (ordinary life situations) will therefore persist as a difficult art. Their main desire focuses on the essence of existence, a necessary desire. The events of any personal history and the views of others threaten and weaken identities, identities that are the first element of a relationship with others.

The possibilities, thanks to the progress of verbs and authorized speeches, to maintain a close correlation between each protagonist for a better life without too many disabling risks (artificial life of seriously ill patients, is a good example) imply the arrival of difficult ethical questions. As a result, there are desperate situations of failure (medical and/or the end of positive vision) where the pursuit of certain protocols becomes unreasonable and leads to what is commonly called stubbornness, whether therapeutic, psychological or even disproportionately out of scope. This senseless determination, often difficult to define, includes, as any freedom of action that begins only where the usefulness of the behaviour ceases, its relevance to its targets; that is, when the continuation of treatment does not allow one to hope for a continuous existence with an acceptable quality of life. Judging the usefulness of a behaviour therefore requires an assessment of an overall prognosis (if treatment is as complete as possible), and as precise a knowledge as possible of what the quality of life accepted or acceptable by the patient or his relatives, including for the staff who will surround him (accompanying), could be. The ethical choices (social and/or medical) in these situations are complex because they depend on many factors based on sometimes conflicting moral values; and questioning above all the very purpose of physical, psychological, medicinal and corollary treatments whose limits become difficult to perceive. "In the absence of a specific role, we sometimes had to take risks. (...) We lived in a poorly defined space. A slightly scary space between dream and reality"2. François Jacob speaks in this way for his first contact with a patient as soon as he is invested as a doctor. Nothing changes in this world except the way of exercising and especially the way of expressing oneself, of seeing the relationships on situations. Ethics in medical action in the field of serious hazards raises all these questions but is characterized by the serious security provided to the mind and direct perceptions including stopping in terms of disability if behavioral nonobservations are issued. However, the plasticity of our brains, taking into account brain damage related to obstruction or rupture, can be responsible for a sudden neurological, mechanical and/or cognitive

${ }^{1}$ Baruch de Spinoza, Ethique, Livre III, Paris, publisher Flammarion, coll. Garnier Philosophie, 1993.

${ }^{2}$ François JACOB, The Inner statue, Paris, publisher O. Jacob, Le Seuil, 1987. 
startle that can lead the patient to extremes quickly or to induce severe and persistent disabilities in the ordinary. The questioning that led to this research work was therefore born from the doubts and difficulties encountered along the way about behavior (legal or medical or simply social) where these particular (Body/Mind) and complex situations lead us to act in an emergency. The bodies (in the future) of the disabled or the disability itself are often considered very uncertain; or even more difficult those of the quality of life of the patient becoming aware of his body and mind. The subjects raised by these situations are numerous and are mainly based on the prognosis and proportionality of care and evaluation. Anticipation determines the decision to initiate, continue or stop certain behaviors, undeniable changes in medication, if the incapacity is very severe or even "unacceptable". The objective is therefore to explore the complexity of these situations to better understand them, and to propose an ethical approach to behavioral or neural medical action in this difficult context. In our overall harmonization of actions, we must define what is called "emergence" as being the vision of a perception resulting from the simultaneous existence of several sources of information, collection not detectable in each of the structures individually examined. The whole system is in fact based on rapid comprehension and intentional actions: the result of increased plasticity of each participant's brain. Also to be wary of any inconsiderate urgency, any emergence is not (cannot be) detectable and predictive certainty only exists in certain cases. To each word, each verbal element appears "virtual" images that complement the phonetics and representations known with these words: what I could call a spectrum. Thus, neural signal models and our knowledge from the syntheses of our behaviors - or only from our perception of sounds, pains-can explain the apparitions, in convincing ways, of phenomenology such as heartbeats (febrile, abundant excitement) or tremors or mental pressures; or consequently bring amplitudes to the complex results of the analysis of behaviors deepening the differentials that may arise ${ }^{3}$. Let us not forget that each representation includes direct decoding and mental decoding, both interfering not in each other but one after the other within agreed millisecond timescales. The emergence of complexity is from this moment perceptible. Studies conducted over the past century or so have provided this understanding of the conditions under which several source factors can "merge" to form a single new perceptual concept; without, however, providing a global response to these new emergences. Emergence goes hand in hand with the unpredictability of which I think that each unit, however exercised, will always be limited in its ability to represent mentally only alloys of images and sounds, alloys of visual representation coupled with diffuse sound representations. The volume of the space becomes too large, offering constraints of understanding, constraints of sound and visual environments. In subsequent explorations, prognostic representations encompassing critical intuition will lead to an understanding and discussion of these ethical issues raised for this "appropriate" medical action proportionate to the overall difficulty exposed. Body and Spirit, a Being of now, hear the essential in two corpora, the visible and the supposed, simultaneously. With the meaning and use of any "prognostic engagement", participants especially want to understand the meaning before any actions, medical or similar initiative. It is complex and even ambiguous that the complexity of an emergence can be understood as a solution and can be at the same time prognostic, prediction and observation of an evolution for the patient, the doctor and health. I say Health, because that is the domain here. Certainly, any debate on the subject is a difficult art, an anticipation aimed at prognosis and possible cure supported by this set characterized by an increasingly scientific situation. This concern of importance for physicians shows that they must be called personnel who initiate a form of care: difficult know-how. It is obvious that this being whose ability to say things is endowed with both behavioral visions. Emergencies and their complexities cannot be evidence of action to others. The factors and models taught or tamed in digital form are necessary without being able to be questioned or controlled until the uncertainties are ignored. These exist inherent in any prediction situation in order to guide a position (medical or other). In all cases of situations of concern, unpredictability should be recognized as somehow forming an essential body of knowledge to be developed, as they become the main justifications for action. Similarly, the medical action in these concordances of proportionality of applications remains complex and qualifies the issue. The use of the term action is justified and "applies to things as different as natural causality, expressive behaviors (laughing, crying, screaming, etc.), instrumental activities (placing, moving, grabbing an object, etc) and acts directed at others"4.

\section{Conclusion}

We must refer to the analyses of a particular (specialized) literature that proposes as a goal the research on prognosis, prediction and immediate installation of a response thanks to an adapted and congruent knowledge. This implies a power of rapid and detailed analysis, followed by decisions that provide the geography of therapeutic limitations; what any knowledge (medical or otherwise) can teach us about these situations. As data on immediate action (social, medical) in the field of neurodegeneration are limited, reference should also be made to the much more numerous studies on therapeutic limitations associated with social and behavioral elements. As we show, the latter shed light on the questions raised by medical action relating to the proportionality of applications and the durability of the effects generated on people. The body places individuals in a world that is as physical as it is intersubjective, social and historical-cultural. We must realize that all organisms are alienated but also alienating. Beings are in permanent cognitive combat with their anatomy and flesh. It is up to us to help control the physical and mental bodies, increasing alertness in the face of stress and suffering that is often invisible. All bodies coexist with and thanks to devices (internal as well as external) and dependencies allow the figurations of capacities of

${ }^{3}$ Psychovisual, psychoacoustic, psychological and psychosensory studies

${ }^{4}$ Ogien Ruwen, Dictionary of Ethics and Moral Philosophy, edited by Monique Canto- Sperber. Paris, publisher PUF, coll. Quadrige, 2004. 
proximities, careers and will amplify them. The consideration of all environmental factors goes far beyond the phenomenology of social and medical ethics, so it is necessary to undertake to bring human development to life as a process through which any person can acquire a broad conception that reasonably evolves his mind.

\section{Acknowledgements}

None.

\section{Conflict of Interest}

No Conflict of Interest. 